\title{
Análisis genético, clínico y molecular de una familia afectada con una malformación del esmalte dental
}

\author{
Blanca U rzúa $0^{\text {1ab }}$, Ana 0 rtega P2cd, Luis Rodríguez Mc, \\ Irene Morales B ${ }^{1 \mathrm{~cd}}$. \\ Genetic, clinical and molecular \\ analysis of a family affected by \\ amelogenesis imperfecta
}

Background: Amelogenesis Imperfecta (AI) is a group of conditions where there is an abnormal formation of enamel in terms of quantity, structure and composition. AI is clinically and genetically heterogeneous, there are sex linked and autosomal versions, dominant and/ or recessive, with phenotypes of hypoplastic, hypocalcified or hypomature enamel. Only recently, through clinical, genetic and molecular studies of affected families, phenotypic-genotypic correlations are being established in this group of anomalies. Aim: To carry out a genetic, clinical and molecular analysis of a Chilean family affected with an enamel malformation, which probably would correspond to Amelogenesis Imperfecta Dominant Autosomal (AIDA), of hypoplastic type, resulting from g.6395G>A mutation in the enamelin gene. Patients and Methods: A genealogical pattern was created for five generations. Five members of this family group were clinically examined, and four of them had a molecular analysis that consisted of the detection of a mutation in the enamelin gene using PCR. Results: In this family, the enamel malformation presents a dominant autosomal pattern of inheritance. The clinical examination of the group allowed a diagnosis of Amelogenesis Imperfecta, of the hypoplastic local type. However, the molecular analysis revealed that the members analyzed did not exhibit the g.6395G >A mutation reported for the enamelin gene (ENAM). Conclusions: The enamel phenotype in this family could be explained by the presence of one of four other mutations recently described in this or another gene, thereby supporting the findings of allelic heterogeneity reported in the literature (Rev Méd Chile 2005; 133: 1331-40).

(Key Words: Amelogenesis imperfecta; Dental enamel hypoplasia; Mutation)

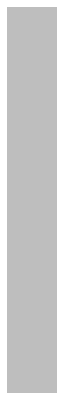

Recibido el 6 de enero, 2005. Aceptado el 13 de junio, 2005.

Trabajo financiado por proyecto PRI-ODO 04/23 de la Facultad de Odontología, Universidad de Chile.

${ }^{1}$ Laboratorio de Bioquímica y Biología Oral, Departamento de Ciencias Físicas y Químicas, Facultad de Odontología, Universidad de Chile.

2Laboratorio de Anatomía Patológica, Departamento de Patología, Facultad de Odontología, Universidad de Chile.

aBióloga

boctor en Ciencias c/m Biología

${ }^{\mathrm{c}}$ Cirujano-Dentista

dMagíster en Patología Oral

Correspondencia a: Dra. Blanca Urzúa O. Laboratorio de Bioquímica y Biología Oral, Facultad de Odontología, Universidad de Chile. Olivos 943, Independencia, Santiago, Chile. Fono: 9781793. Fax: 9781808. E-mail: burzua@uchile.cl 
$\mathrm{E}$ esmalte es un tejido de origen ectodérmico, altamente mineralizado, que no puede ser reparado, ya que los ameloblastos que le dan origen, se pierden durante la erupción del diente ${ }^{1}$. Es sintetizado en el período de odontogénesis, en la séptima semana de gestación, y continúa durante años después del nacimiento ${ }^{2}$.

En la etapa secretora de la amelogénesis, se produce una matriz proteica, mineralizada en $30 \%$, correspondiente a todo el grosor del esmalte adulto $^{3}$. En la etapa madurativa, se degrada esta matriz y se completa la mineralización. La matriz de la etapa secretora está formada por $90 \%$ de amelogeninas y $10 \%$ de proteínas no amelogeninas ${ }^{4}$. Entre éstas últimas se distinguen tuftelina, enamelina y ameloblastina ${ }^{5}$. También se sintetizan proteasas; en la etapa secretora predominan las metaloproteasas, como enamelisina (MMP-20) ${ }^{6}$, y en la etapa madurativa las proteasas de serina como calicreína 4 (EMSP1) ${ }^{7}$.

Las únicas patologías hereditarias que afectan el esmalte, son las amelogénesis imperfectas $(\mathrm{AI})^{8}$, un grupo de condiciones en las que hay formación de esmalte anormal en cantidad, estructura, composición o ambas ${ }^{9}$. Así, los principales genes candidatos para mutaciones causales en AI son aquellos que codifican proteínas involucradas en la ruta de síntesis de esmalte.

La prevalencia de AI varía desde 1 en 700 en Suecia, a 1 en 14.000 en Estados Unidos ${ }^{10,11}$. En nuestra población, no hay estudios que detallen los subtipos de este desorden y su prevalencia.

AI es clínica y genéticamente heterogénea. Según las distintas etapas de la amelogénesis afectada, clínicamente se observan AI hipoplásicas, hipocalcificadas e hipomaduras, existiendo combinaciones de ellas. La hipoplasia puede presentarse como lisa, rugosa, localizada, generalizada o con hoyos. El tipo hipomaduro como pigmentado o con dientes en gorro de nieve. El fenotipo puede variar dentro de una familia, entre familias, entre individuos de una misma familia y entre piezas dentarias de un mismo individuo, presentándose en la dentición temporal, permanente o ambas. Genéticamente, es transmitida en forma autosómica dominante, recesiva o ligada al cromosoma $\mathrm{X}^{12-14}$.

Actualmente, mediante el estudio clínico y genético-molecular de familias afectadas, se están estable- ciendo comelaciones fenotipo-genotipo en $\mathrm{AI}^{15-18}$. Las ligadas a X serían causadas por 14 mutaciones en el gen de amelogenina (AMELX) ${ }^{19-30}$. Para las AI de herencia autosomal, se ha reportado una mutación en el gen de calicreína 4 (KLK4) causante de una variedad de AI recesiva hipomadura ${ }^{31}$ y una mutación en el gen de MMP-20 responsable de AI recesiva hipomadura pigmentada ${ }^{32}$. En el gen ENAM se han descrito cinco mutaciones; la primera corresponde a la variante g.6395G >A asociada con amelogénesis imperfecto dominante autosomal (AIDA) hipoplásica, lisa, generalizada ${ }^{33}$. La variante g.2382A >T es causal de AIDA hipoplásica local, con hoyos y grandes áreas de esmalte afectado ${ }^{34}$. La mutación g.8344delG se asocia con AIDA hipoplásica lisa ${ }^{15,35}$ y con AIDA hipoplásica generalizada ${ }^{36}$. La variante g.13185_13186insAG, en estado homocigoto causa un fenotipo de AI recesiva autosomal (AIRA) hipoplásica generalizada, asociada a mordida abierta anterior (MAA). En heterocigosis el fenotipo es dominante para defectos del esmalte hipoplásico localizado (DEHL) ${ }^{37}$. Recientemente, se reportó la quinta mutación, g.4806A $>$, responsable de AIDA hipoplásica generalizada, rugosa, y se describió una nueva expresión clínica de la variante de deleción, esta vez asociada con hipoplasia generalizada ${ }^{36}$.

En nuestro laboratorio, estamos interesados en aportar al conocimiento de la síntesis de esmalte, a través del estudio de familias con esta condición, para identificar mutaciones en los genes involucrados, lo que incidirá directamente en los criterios de clasificación y prevención.

El objetivo de este trabajo es realizar un análisis genético, clínico y molecular de una familia chilena afectada con una malformación del esmalte, que correspondería a AIDA, de tipo hipoplásica, probablemente producida por la mutación g.6395G $>A$, en el gen de enamelina.

\section{PACIENTES Y MÉTODO}

\section{Análisis genético}

Pacientes y controles. Cinco integrantes de la familia, que dieron su consentimiento informado, fueron examinados clínicamente. Se incluyó tres sujetos controles no relacionados, sin amelogénesis. La probando relató la historia familiar y se 
construyó el patrón genealógico usando el programa PROGENY 538. En los casos en que no se realizó examen clínico en consulta, se asignó el fenotipo por inspección visual y entrevista a cargo de un alumno interno. La determinación del patrón de transmisión, se realizó por un análisis tradicional de la genealogía, tomando en cuenta los criterios para cada tipo de herencia.

\section{Análisis clínico}

Anamnesis. Se consultó por historia de enfermedades sistémicas, tratamiento farmacológico o antecedentes que pudieran afectar la estructura dentaria durante el desarrollo. Se consultó por odontalgia frente a estímulos térmicos y químicos.

Examen clínico extra e intra oral. Se realizó en una consulta odontológica, con espejo № 5 y sonda de caries. Se consignaron datos relativos a MAA, dentición afectada, alteraciones de forma, tamaño y color de los dientes. La clasificación de AI se realizó según Witkop ${ }^{39}$.

Análisis fotográfico y radiográfico. Los pacientes fueron fotografiados con una cámara digital (Fujifilm, Fine Pix S602 Zoom). Se tomaron radiografías retroalveolares, con un equipo de rayos $\mathrm{X}$ de $70 \mathrm{~Hz}$ (Dental $\mathrm{X}$, rayos ARM modelo dx-076, Belmont $\left.{ }^{\circledR}\right)$, las que se analizaron en negatoscopio convencional.

\section{Análisis Molecular}

Purificación de ADN genómico. El ADN genómico de pacientes y sujetos controles se purificó usando el sistema comercial PROMEGA ${ }^{\circledR}$, siguiendo las instrucciones del fabricante.

Reacción en cadena de la polimerasa (RCP). Se amplificó un sector del gen ENAM, en la vecindad exón- intron 8, utilizando partidores cuya secuencia fue obtenida de Rajpar33. La RCP se realizó en un termociclador Perkin Elmer $^{\circledR}$, modelo 2400. Como molde se usó 50-100 ng de ADN. Cada reacción, de $25 \mu \mathrm{l}$, contenía: tampón PCR 10X pH 8.4, $2 \mathrm{mM}$ de $\mathrm{MgCl}_{2}, 0,2 \mathrm{mM}$ de desoxirribonucleótidos, $1 \mu \mathrm{M}$ de cada partidor y 0,625 unidades de polimerasa. Las condiciones de reacción fueron: $95^{\circ} \mathrm{C}$ por $5 \mathrm{~min}$, seguido por 35 ciclos a $95^{\circ} \mathrm{C}$ por $30 \mathrm{~s}$, alineamiento a $55^{\circ} \mathrm{C}$ por 30 s y extensión a $72^{\circ} \mathrm{C}$ por $3 \mathrm{~min}$, con un paso de extensión final a $72^{\circ} \mathrm{C}$ por $10 \mathrm{~min}^{33}$.
Electroforesis. Las muestras de la RCP fueron chequeadas mediante electroforesis en geles de poliacrilamida al $12 \%$, en tampón TBE $1 \mathrm{X}$ y visualizadas por tinción con bromuro de etidio ${ }^{40}$.

Purificación y digestión del fragmento de 214 pb. El amplicón de $214 \mathrm{pb}$ fue purificado por el método de unión a sílica ${ }^{41}$, chequeado por electroforesis y digerido totalmente con la enzima HhpI, durante $12 \mathrm{~h}$ a $37^{\circ} \mathrm{C}$.

\section{RESULTADOS}

Análisis genético. En la Figura 1A, se observa transmisión vertical de la malformación, que se expresa fenotípicamente en las generaciones II, III y IV. En todo el patrón genealógico y en estas generaciones el número de hombres y mujeres afectados es aproximadamente igual. Es posible observar transmisión de padre a hijo (desde II.3 a III.5 y III.9 y desde III.5 a IV.6), y de padre a hija (desde III.5 a IV.7 y IV.8). Estos antecedentes sugieren un patrón de herencia autosómica dominante. El individuo II.2 fallecido, estaba probablemente afectado, puesto que su hija (III.2) está afectada con AI y su esposo vivo (II.1) no. En el examen clínico el individuo V.1, de 7 años de edad, en primera fase de la dentición mixta, no evidenció signos de la malformación que aqueja a sus parientes. Los individuos IV.6 y IV.7 están severamente afectados, no así la probando IV.8 (señalada con cabeza de flecha en la Figura 1A) y su padre III.5 quienes están levemente afectados.

Análisis clínico. Durante la anamnesis, no se detectó afecciones locales o sistémicas. La AI se manifestó en la segunda dentición y ninguno de los pacientes presentó MAA. La Figura 1B muestra una fotografía del sujeto III.5, con molares inferiores derechos de coloración blanco opaco. Además, se aprecia parcialmente esmalte delgado en piezas anteriores y menor grosor de esmalte en bordes mesial y distal. La Figura 2A muestra una fotografía del individuo IV.7, con un fenotipo severamente afectado, prácticamente toda la dentición permanente inferior se encuentra comprometida con extensas exposiciones dentinarias. Sus dientes superiores habían sido restaurados antes 
del examen. La Figura 2B, un acercamiento de la fotografía anterior, muestra el esmalte de la pieza 30 de color blanco opaco y los premolares con exigua capa de esmalte. Los molares superiores de esta paciente presentaban el tercio cervical remanente de las cúspides palatinas con esmalte adelgazado, rugoso y con puntos de poca profundidad (no mostrado). La Figura 3A muestra una fotografía del sujeto IV.6, con dientes anteriores,

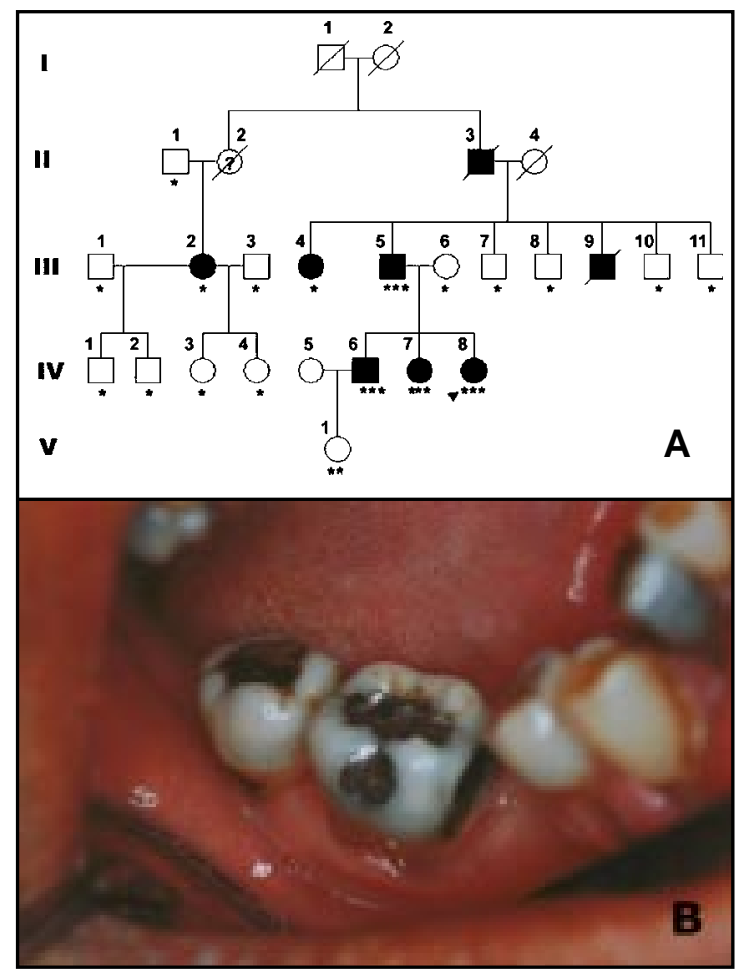

Figura 1A. Patrón genealógico de la familia estudiada, con una malformación del esmalte dental, que podría corresponder a amelogénesis imperfecta (AI). Los círculos y cuadrados negros indican mujeres y hombres afectados con el rasgo, respectivamente. La cabeza de flecha negra señala al probando del estudio. Doble asterisco indica a los individuos que fueron examinados clínica y molecularmente, y un asterisco indica a aquellos examinados sólo clínicamente.

FIgURA 1B. Fotografía clínica del paciente III.5, sexo masculino, 68 años de edad, padre de la probando. Nótese el esmalte blanco opaco presente en los molares. premolares y molares inferiores de coloración blanquecina opaca. Entre los incisivos se observan diastemas y zonas con falta de esmalte en superficies vestibulares. La Figura 3B muestra una fotografía de la probando, donde se aprecia canino y premolares inferiores con falta de esmalte en tercio inferior, que ocasiona surcos horizontales. Además, presentaba diastemas entre lateral, canino y premolar por escasez de esmalte, la cual fue más marcada en canino (no mostrado).

Análisis radiográfico. Las radiografías de la paciente IV.7, mostradas en la Figura 4A y 4B,

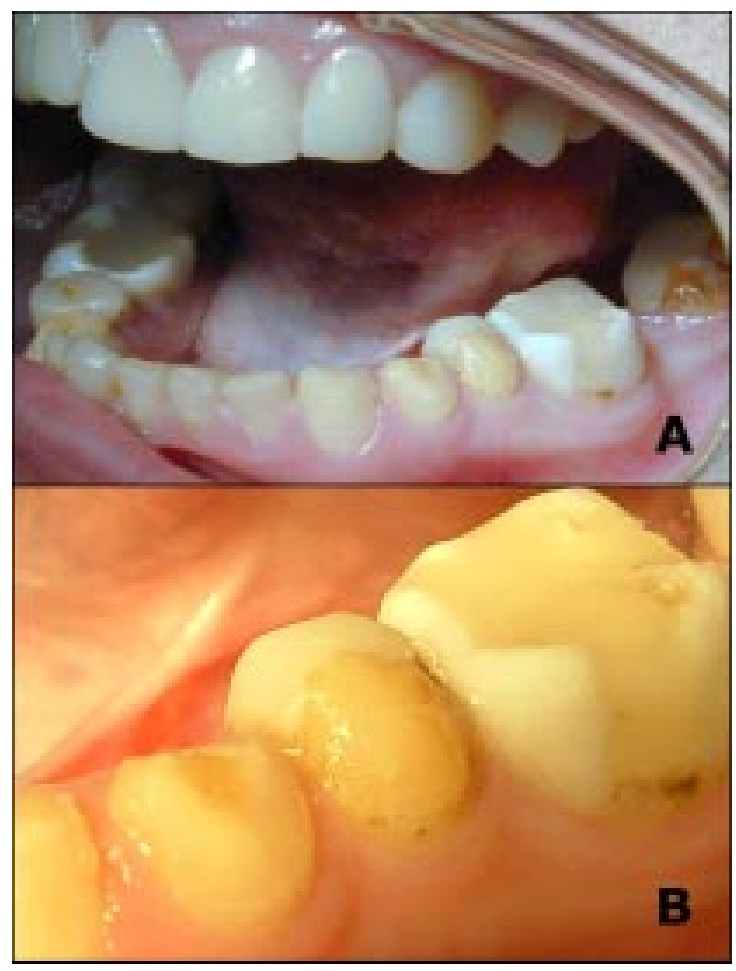

FiguRA 2A. Fotografía clínica de la paciente IV.7, sexo femenino, 28 años de edad, hermana de la probando. Obsérvese la extensión de las áreas hipoplásicas en dientes inferiores, en donde, desde los premolares hacia mesial, el esmalte es muy delgado.

FIgURA 2B. Fotografía clínica de la paciente IV.7, mostrando una visión aumentada de las piezas 28, 29 y 30 . Obsérvese la pieza 30 con esmalte blanquecino y las piezas 28 y 29 esmalte adelgazado. 


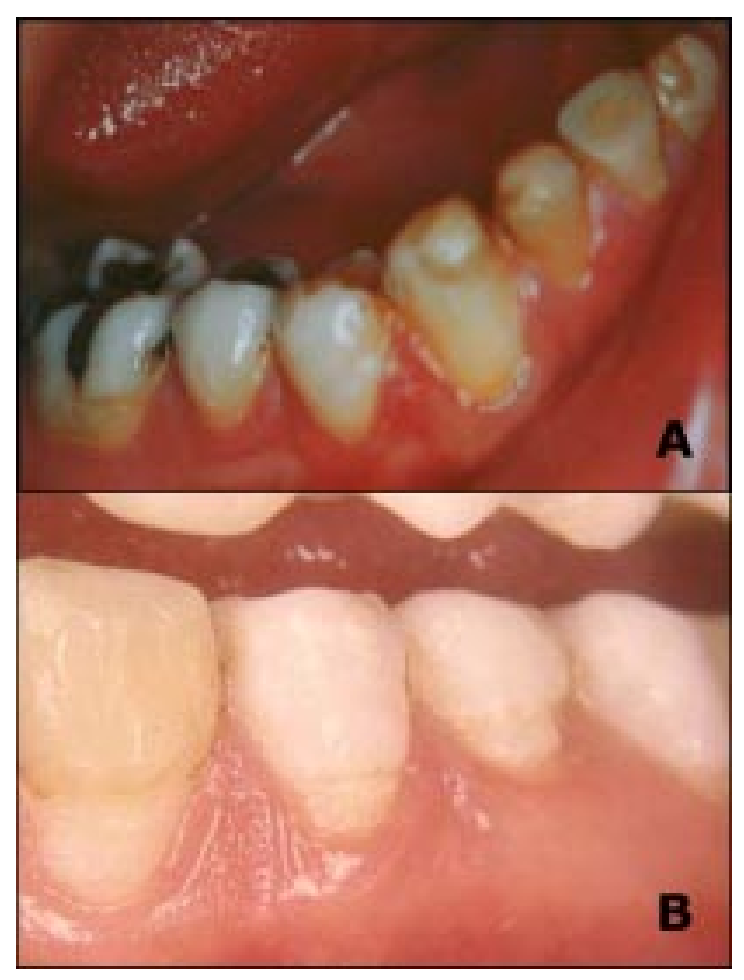

FIgURA 3A. Fotografía clínica del paciente IV.6, sexo masculino, 30 años de edad, hermano de la probando. Se observan dientes antero inferiores y premolares con áreas hipoplásicas y desgaste incisal pronunciado. FIgURA 3B. Fotografía clínica del paciente IV.8, mujer de 24 años, probando. Canino y premolares inferiores con hipoplasias cervicales.

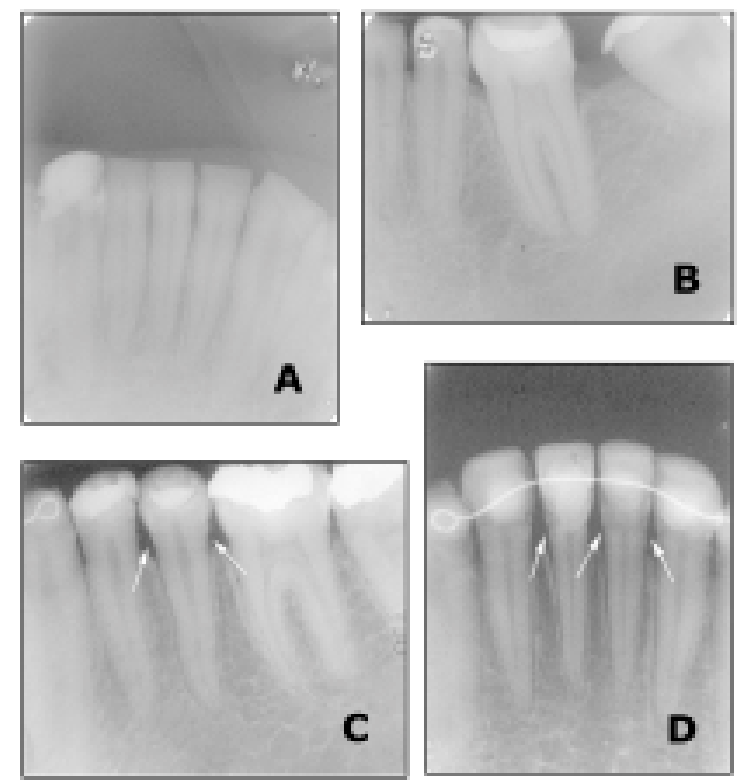

FIgURA 4A. Radiografía retroalveolar periapical de las piezas antero inferiores de la paciente IV.7. Nótese la delgadez del esmalte y la falta de contraste entre éste y la dentina subyacente.

FiguRA 4B. Radiografía retroalveolar periapical de las piezas 29, 30 y 32 de la paciente IV.7. Se observa la escasez de esmalte por toda la corona de la pieza 29, por distal de la pieza 30 y por mesial de la pieza 32 .

FIGURA 4C. Radiografía retroalveolar periapical de las piezas inferiores de la paciente IV.8. Obsérvese el menor grosor del esmalte en el tencio cervical, formándose esta especie de rodete que circunscribe las piezas mostradas, el cual se destaca en la pieza 29 (flechas).

FIgURA 4D. Radiografía retroalveolar periapical de la zona incisiva inferior de la paciente IV.8. Se destacan (flechas) los incisivos con rodetes cervicales y falta de puntos de contacto. 
revelan la estrecha banda de esmalte en los incisivos inferiores y premolares. También se aprecia la falta de contraste entre el esmalte y la dentina subyacente, con ausencia de puntos de contacto. En la Figura 4B se muestra la escasez de esmalte por toda la corona de la pieza 29. Por distal de la pieza 30 se observa esmalte delgado y una fina capa por mesial de la pieza 32. Las radiografías de las Figuras $4 \mathrm{C}$ y $4 \mathrm{D}$, corresponden a la paciente IV.8. Se aprecian bandas horizontales en el tercio cervical coronario de los dientes incisivos y premolares inferiores, con esmalte menos radiopaco. Clínicamente, estas alteraciones se expresan en los contornos laterales mesiales y distales, como áreas de menor grosor o surcos hipoplásicos (ver además Figura 3B). En la Figura $4 \mathrm{D}$ se aprecian claramente los rodetes cervicales que circunscriben a los dientes, con ausencia de puntos de contacto.

La ausencia de alteraciones dietarias o enfermedades sistémicas durante la odontogénesis de los dientes permanentes, la ocurrencia de lesiones en varias generaciones, el compromiso de piezas dentarias con desarrollo cronológico distinto, por ejemplo de incisivos, caninos y premolares, sin afección de primeros molares en un mismo individuo, descartan la posibilidad de alteraciones dentarias ambientales. Por lo tanto, los antecedentes clínicos y el análisis genético, nos conducen al diagnóstico de amelogénesis imperfecta hipoplásica local, de severidad y extensión variable, autosómica dominante, tipo IB, según Witkop ${ }^{39}$.

Análisis molecular. Para comprobar la existencia de la mutación descrita por Rajpar33, los productos RCP de $214 \mathrm{pb}$ provenientes de sujetos controles y pacientes, fueron purificados y posteriormente digeridos con la enzima HphI. Esta enzima corta el fragmento de $214 \mathrm{pb}$ en dos; uno de $116 \mathrm{pb}$ y otro de 98 pb. La mutación es heterocigota y suprime el sitio HphI, de tal manera que un heterocigoto afectado debiera presentar tres bandas: una de 214, otra de 116 y una de 98 pb. En la Figura 5 se observa que en los sujetos controles y afectados, los fragmentos de $214 \mathrm{pb}$, digeridos con la enzima, producen bandas de 116 y 98 pb solamente, lo cual significa que el sitio HphI está presente en ambos alelos y por lo tanto estos sujetos no tienen la mutación en el gen de enamelina.

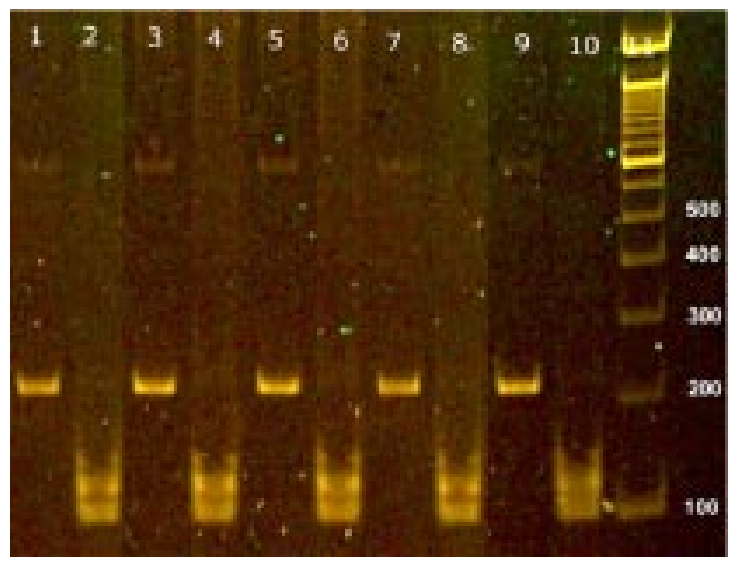

FIgURA 5. Electroforesis en gel de poliacrilamida al $12 \%$, que muestra el análisis realizado para detectar la mutación (g.6395G >A) en la familia estudiada. Canales 1, 3, 5, 7 y 9: fragmento de ADNg de 214 pb intacto, sin digerir, de los pacientes III.5, IV.8, IV.7, IV.6 y sujeto control 2, respectivamente, purificado por el método de unión a sílica. Canales 2, 4, 6, 8 y 10: fragmento de ADNg de $214 \mathrm{pb}$ purificado de los pacientes III.5, IV.8, IV.6, IV.7 y sujeto control 2, respectivamente, digerido con la enzima de restricción HphI. Canal 11: $500 \mathrm{ng}$ de ADN del marcador de tamaño molecular escalera de 100 bp (ladder 100 pb).

\section{DISCUSIÓN}

El análisis clínico permitió observar variaciones en el fenotipo no reportadas; nos referimos al color blanco opaco del esmalte visto en los primeros molares de los pacientes III.5, IV.6 y IV.7. La hipoplasia en esta familia es compatible con lo descrito en la literatura, en relación con la delgadez del esmalte, presencia de surcos y fisuras en molares, premolares e incisivos, con pérdida local de esmalte, más tinciones pardas en algunas zonas. La expresión del fenotipo es más bien localizada, de severidad variable, ya que los individuos IV.6 y IV.7 están más afectados que los sujetos III.5 y IV.8, y de extensión variable, pues el sujeto III.5 presenta afectado molares inferiores derechos y piezas anteriores, en cambio el sujeto 
IV.7 presenta un fenotipo severamente afectado que involucra a toda la dentición inferior y probablemente la superior, lo que en un principio nos condujo a pensar en AIDA generalizada.

Aunque algunas de las clasificaciones consideran el modo de herencia, ninguna de ellas correlaciona el fenotipo con el genotipo, debido a que el estudio molecular comenzó en los años noventa. Esta carencia dificultó la elección del gen candidato y el probable sitio de mutación dentro de él. Por ejemplo, el defecto en nuestra familia correspondería a AIDA hipoplásica en las clasificaciones clínicas más usadas $^{39,42}$. Sin embargo, este fenotipo, que aparece como subtipo local en la clasificación de Witkop ${ }^{39}$, es denominado rugosa con bandas horizontales en la clasificación de Sundell $^{43}$. Estos hechos, la expresividad variable, más las múltiples restauraciones dentarias que enmascaran los rasgos durante el examen, dificultan la aplicación de las clasificaciones basadas en el fenotipo, como la última de Witkop ${ }^{39}$, que siendo la más usada, hoy, sólo sirve como aproximación clínica inicial. Dada la expresividad variable, en extensión y severidad, reportada en varias familias, el estudio molecular debía contemplar aquellas mutaciones causales de AIDA hipoplásica, como la primera variante descrita, g.6395G $>A^{33}$. Estudios posteriores ${ }^{34,35}$ y recientes ${ }^{15,36,37}$ han puesto de manifiesto fenotipos más similares a los encontrados en nuestra familia. La Tabla 1 resume las mutaciones, hasta la fecha descritas para el gen ENAM y los fenotipos clínicos asociados. Sólo, producto de nuestros resultados clínicos y de los recientes reportes resultan ahora obvias, las discrepancias en los fenotipos en nuestra familia y en la estudiada por Rajpar $^{33}$, que manifestó hipoplasia generaliza$\mathrm{da}$, en ambas denticiones y sin expresión variable.

El estudio molecular de nuestro trabajo muestra que, la mutación g.6395G >A no está presente en los afectados, ni tampoco en los controles. Además, solamente después de la visión global de las mutaciones descritas hasta hoy en ENAM
(Tabla 1), es posible pensar en un probable sitio alternativo de mutación.

Tal como se muestra en la Tabla 1 , Kida ${ }^{35}$ observó expresión variable en una familia con la variante g.8344delG, encontrando lesiones horizontales localizadas en el tercio medio de caninos y premolares inferiores, asociada en algunos individuos a sensibilidad térmica y MAA. En otro grupo familiar con la misma mutación, Hart ${ }^{15}$ describe filas horizontales de hoyos en algunas personas y dientes con hipoplasia lisa en otros. Similar, pero no idéntico, fenotipo y expresión variable ha sido recientemente descrita por $\mathrm{Kim}^{36}$. La variabilidad en extensión y severidad de la expresión clínica en estos reportes, son más semejantes a lo encontrado por nosotros. Por estas razones, la hipótesis alternativa, con la que trabajamos actualmente en nuestro laboratorio, es que nuestra familia sería portadora de la variante g.8344delG, pues un fenotipo asociado a ésta presenta esmalte hipoplásico, que afecta localizadamente el tercio medio de la corona en algunos dientes ${ }^{35,36}$. De no ser así, podría tratarse de otra de las mutaciones reportadas ${ }^{34,37}$ o bien una mutación alélica. Incluso podría existir una mutación en otro gen ${ }^{31,32}$, sosteniendo los hallazgos de heterogeneidad alélica y de locus reportados.

La Tabla 1 muestra que se desconoce los valores de frecuencia de casi todas las variantes, excepto el caso del exón 5. Sólo se han comunicado frecuencias para polimorfismos de nucleótidos simples (SNP's) ${ }^{18,34,37}$. Además, es tercera vez que se describe la variante g.8344delG, en familias de diverso origen; Japón ${ }^{35}$, Líbano ${ }^{15}$ e Irán ${ }^{36}$. Sugiriendo que la vecindad exón-intrón 9 representa un sitio caliente de mutación en el gen $\mathrm{ENAM}^{15,36}$, lo que explicaría en parte nuestros resultados y fortalece la hipótesis alternativa. La explicación de los diversos fenotipos intrafamiliares observados por nosotros y varios otros autores no es clara ${ }^{15,35-37}$ y es altamente probable que, junto a las diversas mutaciones de enamelina, la severidad y extensión de AI, esté determinada por otros factores relacionados con la formación del esmalte. 
Tabla 1. M utaciones reportadas en el gen de enamelina (EN AM ) que producen AI con sus fenotipos clínicos observados

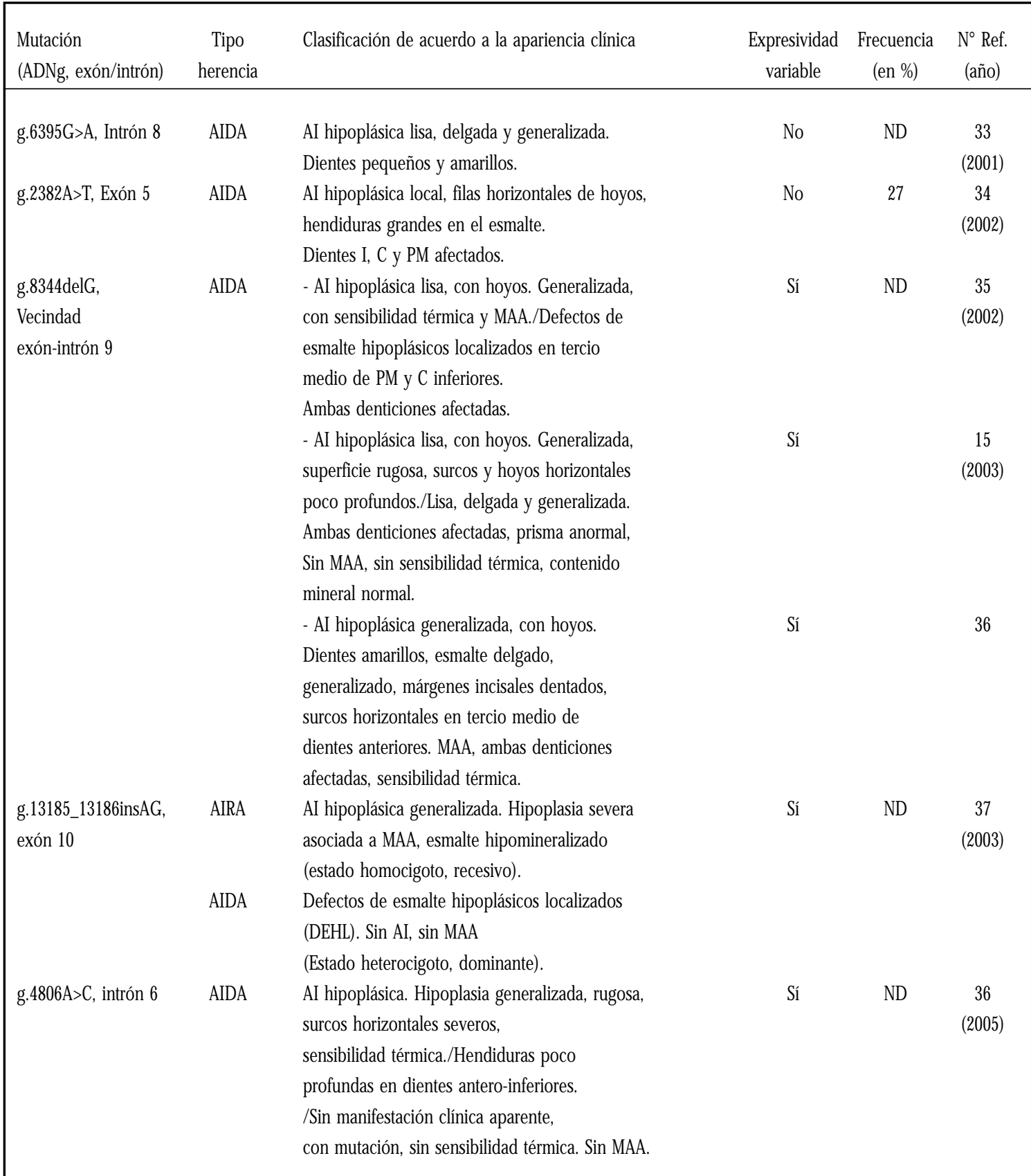

AIDA= Amelogénesis Imperfecta Dominante Autosomal, AIRA= Amelogénesis Imperfecta Recesiva Autosomal, $\mathrm{I}=$ Incisivo, $\mathrm{PM}=$ Premolares, $\mathrm{C}=$ Caninos, $\mathrm{MAA}=$ mordida abierta anterior, $\mathrm{ND}=$ no determinado $/=$ fenotipos clínicos diferentes. 


\section{REFERENCIAS}

1. Ten Cate AR. Oral Histology. Development, structure, and function. Editorial Mosby, 1998; 197217.

2. Gómez de Ferraris ML, Campos A. Histología y embriología bucodentaria. Editorial Médica Panamericana 2002; 271-315.

3. Simmer JP, Fincham AG. Molecular mechanism of dental enamel formation. Crit Rev Oral Biol Med 1995; 6: 84-108.

4. BRoOKes SJ, Robinson C, KirKham J, Bonass WA. Biochemistry and molecular biology of amelogenin proteins of developing dental enamel. Archs Oral Biol 1995; 40: 1-14.

5. Robinson C, Brookes SJ, Shore RC, Kirkham J. The developing enamel matrix: nature and function. Eur J Oral Sci 1998; 106 (suppl 1): 282-91.

6. Bartlett JD, Simmer JP, Xue J, Margols HC, Moreno EC. Molecular cloning and mRNA tissue distribution of a novel matrix metalloproteinase isolated from porcine enamel organ. Gene 1996; 183: 123-8.

7. Simmer JP, Fukae M, Tanabe T, Yamakoshi YM, UChiDA T, XUE J ET AL. Purification, characterization and cloning of enamel matrix serine proteinase 1. J Dent Res 1998; 77: 377-86.

8. Neviue B, Damm D, Auen C, Bouquot J. Oral and maxillofacial pathology. Editorial W.B. Saunders Company 1995; 49-89.

9. Sapp P, Eversole L, Wysocki G. Patología oral y Maxilofacial Contemporánea. Editorial Harcourt Brace, España, 1998; 1-37.

10. Aldred MJ, Savarirayan R, Crawford PJM. Amelogenesis imperfecta: a classification and catalogue for the 21 st century. Oral Dis 2003; 9: 19-23.

11. Bäckman B, Holmgren G. Amelogenesis imperfecta: A genetic study. Hum Hered 1988; 38: 189206.

12. Aldred MJ, Crawford PJM, Roberts E, Gilespie CM, Thomas NST, Fenton I et aL. Genetic heterogeneity in X-linked amelogenesis imperfecta. Genomics 1992; 4: 567-73.

13. Lagerström-Fremér M, Dhal N, Iseluus L, Bäckman B, PetTersson U. Mapping of the gene for X-linked amelogenesis imperfecta by linkage analysis. Am J Hum Genet 1990; 46: 120-5.

14. Saldio EC, Yen PH, Koprivnikar K, Yu L-C, Shapiro LJ. The human enamel protein gene amelogenin is expressed from both the $\mathrm{X}$ and the $\mathrm{Y}$ chromosomes. Am J Hum Genet 1992; 50: 303-16.
15. Hart PS, Michalec MD, Seow WK, Hart TC, Wright JT. Identification of the enamelin (g.8344delG) mutation in new kindred and presentation of standardized ENAM nomenclature. Archs Oral Biol 2003; 48: 589-96.

16. Nusier M, Yassin O, Hart TC, Samimi A, Wright JT. Phenotypic diversity and revision of the nomenclature for autosomal recessive amelogenesis imperfecta. Oral Surg Oral Med Oral Pathol Oral Radiol Endod 2004; 97: 220-30.

17. Wright JT, Hart PS, Aldred MJ, Seow K, Crawford PJM, Hong SP ET AL. Relationship of phenotype and genotype in X-linked amelogenesis imperfecta. Connect Tissue Res 2003; 44 (Suppl): 72-8.

18. Hu JC-C, Yamakoshi Y. Enamelin and autosomaldominant amelogenesis imperfecta. Crit Rev Oral Biol Med 2003; 14: 387-98.

19. Aldred MJ, Crawford PJM, Roberts E, Thomas NST. Identification of a nonsense mutation in the amelogenin gene (AMELX) in a family with Xlinked Amelogenesis imperfecta (AIH1). Hum Genet 1992; 90: 413-6.

20. Cower PM, Sauk JJ, Rosenbloom J, Yuan ZA, Gibson CW. An amelogenin gene defect associated with human X-linked amelogenesis imperfecta. Archs Oral Biol 1997; 42: 235-42.

21. HaRt S, HaRT T, GibSon C, Wright JT. Mutacional analysis of X-linked amelogenesis imperfecta in multiple families. Archs Oral Biol 2000; 45: 79-86.

22. Hart PS, HaRT TC, Simmer JP, Wright JT. A nomenclature for X-linked amelogenesis imperfecta. Arch Oral Biol 2002; 47: 255-60.

23. Hart PS, Aldred MJ, Crawford PJM, Wright NJ, HART TC, Wright JT. Two amelogenin gene mutations cause different amelogenesis imperfecta phenotypes. Arch Oral Biol 2002; 47: 261-5.

24. Kindelan SA, Brook AH, Gangemi L, Lench N, Wong FSL, FEARNE J ET AL. Detection of a novel mutation in X-linked amelogenesis imperfecta. J Dent Res 2000; 79: 1978-82.

25. LAgerström-Fermér M, Dhal N, NAKAhori $Y$, MAKAgome Y, Bäckman B, LANDEgREN U et AL. A deletion in the amelogenin gene (AMG) causes X-linked Amelogenesis imperfecta (AIH1). Genomics 1991; 10: 971-5.

26. Lagerström-Fermér M, NiLSSON MBJ, BäcKMAN B, Shapiro LJ, Pettersson U, Landegren U. Amelogenin signal peptide mutation: Correlation between mutation in the amelogenin gene (AMGX) and manifestations of X-linked Amelogenesis imperfecta. Genomics 1995; 26: 159-62. 
27. Lench NJ, Brook AH, WinTER GB. SSCP detection of a nonsense mutation in exon 5 of the amelogenin gene (AMGX) causing X-linked amelogenesis imperfecta (AIH1). Hum Mol Genet 1994; 3: 827-8.

28. Lench NJ, Brook AH. DNA diagnosis of X-linked amelogenesis imperfecta (AIH1). J Oral Pathol Med 1997; 26: 135-7.

29. Ravassipour DB, Hart PS, Hart TC, Ritter AV, YAMAUCHI M, GiBSON C ET AL. Unique enamel phenotype associated with amelogenin gene (AMELX) codon 41 point mutation. J Dent Res 2000; 79: 1476-81.

30. Kim J-W, Simmer JP, Hu YY, Lin BP-L, Boyd C, Wrigth JT ET AL. Amelogenin p.M1T and p.W4S mutations underlaying hipoplastic X-linked amelogenesis imperfecta. J Dent Res 2004; 83: 378-83.

31. HaRT PS, Hart TC, Michalec MD, Ryu OH, Simmons D, Hong S ET AL. Mutation in kallikrein 4 causes autosomal recessive hypomaturation amelogenesis imperfecta. J Med Genet 2004; 41: 545-9.

32. Kim J-W, Simmer JP, Hart TC, Hart PS, Ramaswami MD, Bartlett JD ET AL. MMP-20 mutation in autosomal recesive pigmented hypomaturation amelogenesis imperfecta. J Med Genet 2005; 42: 271-5.

33. Rajpar HM, Hariey K, Laing C, Davies RM, Dixon MJ. Mutation of the gene encoding the enamelspecific protein, enamelin, causes autosomaldominant amelogenesis imperfecta. Hum Mol Genet 2001; 10: 1673-7.

34. MARdh CK, Bäckman B, Holmgren G, Hu JC-C, SiMMER JP, FörSMAN-SEMB K. A nonsense mutation in the enamelin gene causes local hypoplastic autosomal dominant amelogenesis imperfecta (AIH2). Hum Mol Genet 2002; 11: 1069-74.

35. Kida M, Ariga T, Shirakawa T, Oguchi H, Sakiyama Y. Autosomal-dominant hypoplastic form of amelogenesis imperfecta caused by an enamelin gene mutation at the exon-intron boundary. J Dent Res 2002; 81: 738-42.
36. Kim J-W, Seymen F, Lin BP-J, Kiziltan B, Gencay K, SiMMER JP ET AL. ENAM mutations in autosomal dominant amelogenesis imperfecta. J Dent Res 2005; 84: 278-82.

37. Hart TC, Hart PS, Gorry MC, Michalec MD, Ryu $\mathrm{OH}$, Uygur C ET AL. Novel ENAM mutation responsible for autosomal recessive amelogenesis imperfecta and localised enamel defects. J Med Genet 2003; 40: 900-6.

38. PROGENY 5 (www.progeny2000.com)

39. Wтткор CJ. Amelogenesis imperfecta, dentinogenesis imperfecta and dentin dysplasia revisited: problems in classification. J Oral Pathol 1989; 17: 547-53.

40. Sambrook JF, Maniatis T. Molecular Cloning: A laboratory manual. Cold Spring Harbor Laboratory Press, 1989; 6.3-6.60.

41. Boyle JS, LEw AM. An inexpensive altemative to glass milk for DNA purification. Trends in Genetics 1995; 11: 6-7.

42. Online Mendelian Inheritance in Man, OMIM (TM). Johns Hopkins University, Baltimore. MIM number: $\{23 / 3 / 2004\}$ : 300391$\}$. World wide web URL: http://www.ncbi.nlm.nih.gov/omim/.

43. SundeLL S, VAlentin J. Hereditary aspects and classification of hereditary amelogenesis imperfecta. Community Dent Oral Epidemiol 1986; 14: 211-6.

Agradecimientos

Se agradece a los pacientes que gentil y desinteresadamente participaron en este estudio y voluntariamente aceptaron ser examinados y donaron una muestra de sangre para el análisis molecular. Agradecemos también, muy especialmente, a la Dra. Marcia Cuevas por derivarnos a la familia en estudio y por toda la colaboración prestada. 